\title{
Application of computed order tracking, Vold-Kalman filtering and EMD in rotating machine vibration
}

\author{
K. S. Wang and P. S. Heyns
}

Dynamic Systems Group, Department of Mechanical and Aeronautical Engineering, University of Pretoria, Pretoria, 0002, South Africa

\begin{abstract}
This paper presents a study on rotating machine vibration signals by using computed order tracking, Vold-Kalman filtering and intrinsic mode functions from the empirical mode decomposition method. Through the sequential use of intrinsic mode function and order tracking methods, both speed synchronous and non-synchronous vibrations that modulate orders in rotating machine vibrations are distinguished, which is difficult when using each of the techniques in isolation alone. Simulation and experimental studies demonstrate the ability of extracting vibrations that modulate order signals through combining the techniques.

Keywords: Computed order tracking (COT), Empirical mode decomposition (EMD), intrinsic mode function (IMF), rotating machinery, Vold-Kalman filter order tracking (VKF-OT).
\end{abstract}




\section{Introduction}

Vibration signals from rotating machinery contain ample information for the diagnosis of machine faults, and it has been widely recognized that vibration signal analysis is an effective tool for condition monitoring. One of the key characteristics of vibration signals from rotating machinery is the strong influence that the rotational speed has on the observed signals. Non-constant rotational speed leads to the non-stationarity of the measured signal, which then becomes difficult to interpret. To deal with this, order tracking techniques have been developed. One of the main advantages of order tracking over traditional vibration monitoring techniques lies in its ability to clearly identify non-stationary vibration data, and to a large extent exclude the influences of the varying rotational speed.

Today there are several different classes of order tracking techniques available. For instance, computed order tracking (COT) uses a re-sampling procedure which leads to an order domain spectrum. This is often an effective order tracking method. However, COT does not have the ability to extract each order signal in the time domain, which in turn disposes of the opportunity to do a detailed study of each order signal. Besides, COT deals with the signal as a whole, and it is easy to neglect small changes in non-dominant signal components in a spectral map dominated by orders. However, it does provide an overall picture of composed signals in terms of orders and this makes it a very useful order tracking technique. Other order tracking techniques, such as Vold-Kalman filter order tracking (VKF-OT) or Gabor order tracking, assume sinusoidal waveforms to extract orders in the time domain. They overcome the shortcoming of the methods based on re-sampling and extract order signals in the time domain. However, this compromises the ability to extract changes in signals other than waves of a sinusoidal nature. In reality, few machine signals are purely sinusoidal. For rotating machinery, order signals are usually modulated by machine fault signals, which deform the original shape of the order waves from their sinusoidal nature. In this sense, order tracking techniques such as VKF-OT are incapable of capturing signals that modulate the order wave. As this might be crucial information for machine condition monitoring, a technique which could capture non-stationary signals that modulate orders in the time domain, would be a useful supplement to the order tracking techniques.

In 1998 Huang and his colleagues [1] proposed the Hilbert-Huang transform (HHT) for analysing non-linear and non-stationary signals. Since its introduction, this technique has proven remarkably effective in various mechanical fault diagnostic applications [2, 3, 4]. HHT includes two procedures: empirical mode decomposition (EMD) and the Hilbert transformation. With EMD, complicated signals are broken down into a finite and often small number of intrinsic mode functions (IMFs) based on the local characteristic time scale of the signal. The IMFs represent a collection of natural oscillatory modes embedded in the signal, ranging from high frequency to low frequency. Originally, IMFs were viewed as mono-components representing some 
intrinsic physical meaning and making sense of instantaneous frequency for further Hilbert transformation. The idea of mono-components is arguable however. Cohen [5] first introduced the term but did not give a clear definition. Narrow band components therefore are adopted as a surrogate for IMFs [6]. This makes the EMD process more like a group of narrow band filters, and the method can therefore be treated as a self-adaptive dyadic filter bank [7] which allows each IMF to be determined by the signal itself, rather than being pre-determined. As such, it has the capability of empirically capturing non-stationary signal changes. Since there are no prior assumptions about the nature of the signals decomposed by EMD, sinusoidal and non-sinusoidally-natured oscillating signals can both be contained in each IMF. Realising that an order wave from a rotating machine can also be treated as a modulated sinusoidal oscillating wave, IMFs may well include order waves.

The literature indicates that both IMFs and order tracking techniques are effective in diagnosing faults in rotating machinery [e.g. 8, 9 and 10]. This suggests investigating the relationship between IMFs and order waves. However, this has not been explored further in the literature. In rotating machinery, vibration signals due to faults such as rotor cracks, looseness, worn-out parts, broken teeth or bearing problems are all closely related to the rotating speed or orders. These machine fault signals will usually modulate dominant order waves into modulated oscillating waves, which contain a rich source of machine fault information. As a matter of fact, for condition monitoring of rotating machinery, detecting and separating this information from the dominant orders is of great importance.

To this end, this paper exploits the relationship between an intrinsic mode function (IMF) and an order wave in rotating machinery, and the use of order tracking methods to assist the further decomposition of the IMF. Sequential application of EMD and order tracking methods makes it possible to clarify IMFs in terms of rotational speed and also provides the ability to separate vibrations that modulate order signals (e.g. machine faults vibrations) which cannot be extracted by the use of any of the two methods in isolation alone. Since through EMD the signals are mixed with order signals into IMFs and through order tracking methods such as VKF-OT, these vibrations may not be properly filtered due to the very likely possibilities of non-strict-synchronous with rotational speed. However, combining two methods may get to this information. In the following, the first part of the paper will discuss the relationship between an IMF and an order waveform. The second part of the paper will demonstrate the advantages of sequential use of two kinds of methods in a numerical example as well as an experimental study in which the proposed methodology extracts vibrations that modulate order signals. 


\section{The relationship between an IMF and an order waveform}

EMD and order tracking are both well-established techniques for diagnosing faults in rotating machinery. The IMFs from EMD represent a collection of time-domain waveforms. Order tracking techniques such as VKF-OT can also extract order waveforms in the time domain. So both techniques can extract useful time-domain vibration signals emitted from rotating machinery, which can be used for fault diagnosis. In the following, the relationship between an IMF and an order is explored. Since the focus of the paper is on rotating machine order signals, their relationship in the order domain is discussed.

Firstly, in essence an order can be described as 'a time varying phasor that rotates with an instantaneous frequency related to the rotational frequency of the reference shaft'. Blough [11] defines an order signal in time domain. Based upon his time domain definition of an order, one may write order signals in polar format expressed as function of phase angle:

$$
x(\theta)=A_{o}(k, \theta) e^{j k \theta}
$$

where $A_{o}(k, \theta)$ is the amplitude of order $k, e^{j k \theta}$ is the unit sinusoidal wave of order $k, k$ is the order of interest and $\theta$ is the phase angle.

Similarly, an IMF can also be written in terms of phase angles as shown in Equation (2) (this equation is based upon work by Yang et al. [12]). In this case the amplitude and frequency modulations are both functions of angle $\theta$ instead of time $t$, i.e. in the order domain. Order domain analysis transforms non-stationary time-domain signals into stationary signals in the angular domain for rotating machinery vibrations. The order signals are therefore periodic per revolution and Fourier series expansion is suitable for the analysis.

Therefore any IMF $d(\theta)$ may be expanded as in Equation (2)

$$
d(\theta)=A_{o}(\theta) \cos \phi_{o}(\theta)=\sum_{n=1}^{\infty} C_{n}(\theta) e^{j n \theta}+R(\theta)
$$

where $A_{o}(\theta)$ and $\cos \phi_{o}(\theta)$ are the amplitude and order modulations in the order domain, $C_{n}(\theta)$ are Fourier coefficients and $R(\theta)$ expresses the non-periodic signals in the order domain. 
When comparing Equations (1) and (2), one should note the following:

- The amplitude component $A_{o}(k, \theta)$ in Equation (1) can be one of the amplitude components $C_{n}(\theta), n \in(1, \infty)$, in Equation (2), and

- A unit sinusoidal component $e^{j k \theta}$ in Equation (1) can be one of the components in $e^{j n \theta}, n \in(1, \infty)$, in Equation (2).

Clearly, an order wave can be a particular waveform contained in the IMF. However equation (2) indicates that an IMF can include signals other than orders. But the converse of Equation (2) may not necessarily hold, i.e. a signal of the form of equation (2) may not necessarily constitute an IMF. This is because only signals that satisfy the definition of Huang et al. [1], qualify as IMFs. If the composition of signals violates the definition, it will be further decomposed into different IMFs. This actually suggests that EMD as a filter bank is selective for each IMF. It is difficult to develop a general rule for this selective character or resolution of each IMF, since there is no universal mathematical equation for the EMD.

However, Feldman [13] analyzed the special and useful case of the decomposition of two harmonics, demonstrating some of the important features of EMD, such as the nature of the resolution for each IMF. He describes an analytical basis for the EMD, and presents a theoretical limiting frequency resolution for EMD to decompose two harmonic tones. This helps to understand the resolution of EMD as filters.

Feldman shows that the frequency and amplitude ratios of two harmonics can be separated into three different groups, to evaluate the resolution of EMD for these harmonics:

- Harmonics with very close frequencies and a small amplitude, where $A_{2} / A_{1} \leq\left(\omega_{1} / \omega_{2}\right)^{2}$ are unsuitable for EMD decomposition.

- Close frequency harmonics where $\left(\omega_{1} / \omega_{2}\right)^{2} \leq A_{2} / A_{1} \leq 2.4\left(\omega_{1} / \omega_{2}\right)^{1.75}$ require several sifting iterations for two harmonics to decompose.

- Distant frequencies and large amplitude harmonics where $A_{2} / A_{1} \geq 2.4\left(\omega_{1} / \omega_{2}\right)^{1.75}$ can be well separated in a single iteration.

Based upon these criteria, one knows that if two harmonics have frequency and amplitude ratios of $A_{2} / A_{1} \leq\left(\omega_{1} / \omega_{2}\right)^{2}$, EMD is incapable of separating them. This 
requirement is visually depicted in Figure 1.

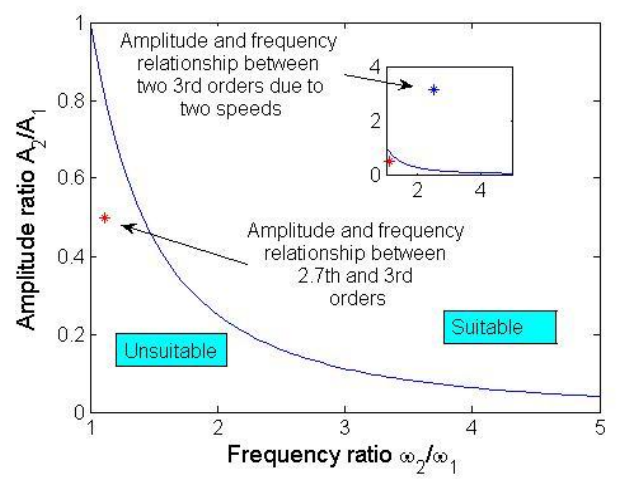

Figure 1: Theoretical boundary for the separation of two harmonics $A_{2} / A_{1} \leq\left(\omega_{1} / \omega_{2}\right)^{2}$

The limiting boundary determines the region to the right of which EMD is able to separate harmonics, and to the left where EMD cannot separate two harmonics [13]. This criterion is very useful for determining the decomposition of harmonic vibration signals like orders in rotating machines.

From the above discussion of the relationship between an IMF and an order, it is clear that IMFs from EMD may include both speed synchronous and non-synchronous vibrations, on the condition that they satisfy the definition of IMF. The EMD process decomposes raw signals into several IMFs and due to this decomposition, different modulated order signals are separated. Considering the intrinsic nature of both order tracking methods and EMD, the IMFs from EMD may be further decomposed in terms of rotational speed through order tracking methods so that speed synchronous and non-synchronous information of an IMF can be distinguished. Consequently, the combined use of EMD and order tracking methods to further decompose order related IMFs is introduced in this paper.

\section{Simulation study}

\subsection{Simulation of a single-degree-of-freedom (SDOF) rotor model}

Linear models of machine faults are valuable and sufficient for many purposes [14]. In the current simulation study, a linear rotor model is therefore considered.

The lateral response of a symmetric rotor is modelled as two uncoupled SDOF systems. It is assumed that a rotor of mass $m$ is mounted on bearings of total stiffness $k$ and damping coefficient $c$. The rotor rotates at an increasing rotational frequency $\omega_{1}$. For details of the model the reader may refer to Wang and Heyns [15] where this model was used to investigate the effects of Vold-Kalman filter bandwidth. 
However, here a more comprehensive external excitation model with superimposed noise on the observed signals is considered. The characteristics of the model are shown in Table 1, considering the response in the vertical y-direction.

\section{Table 1: Simulation model characteristics of the SDOF rotor}

To clarify the composition of observed signals, the signal composition is categorized as speed synchronous and non-synchronous vibrations which are depicted in Figure 2 .

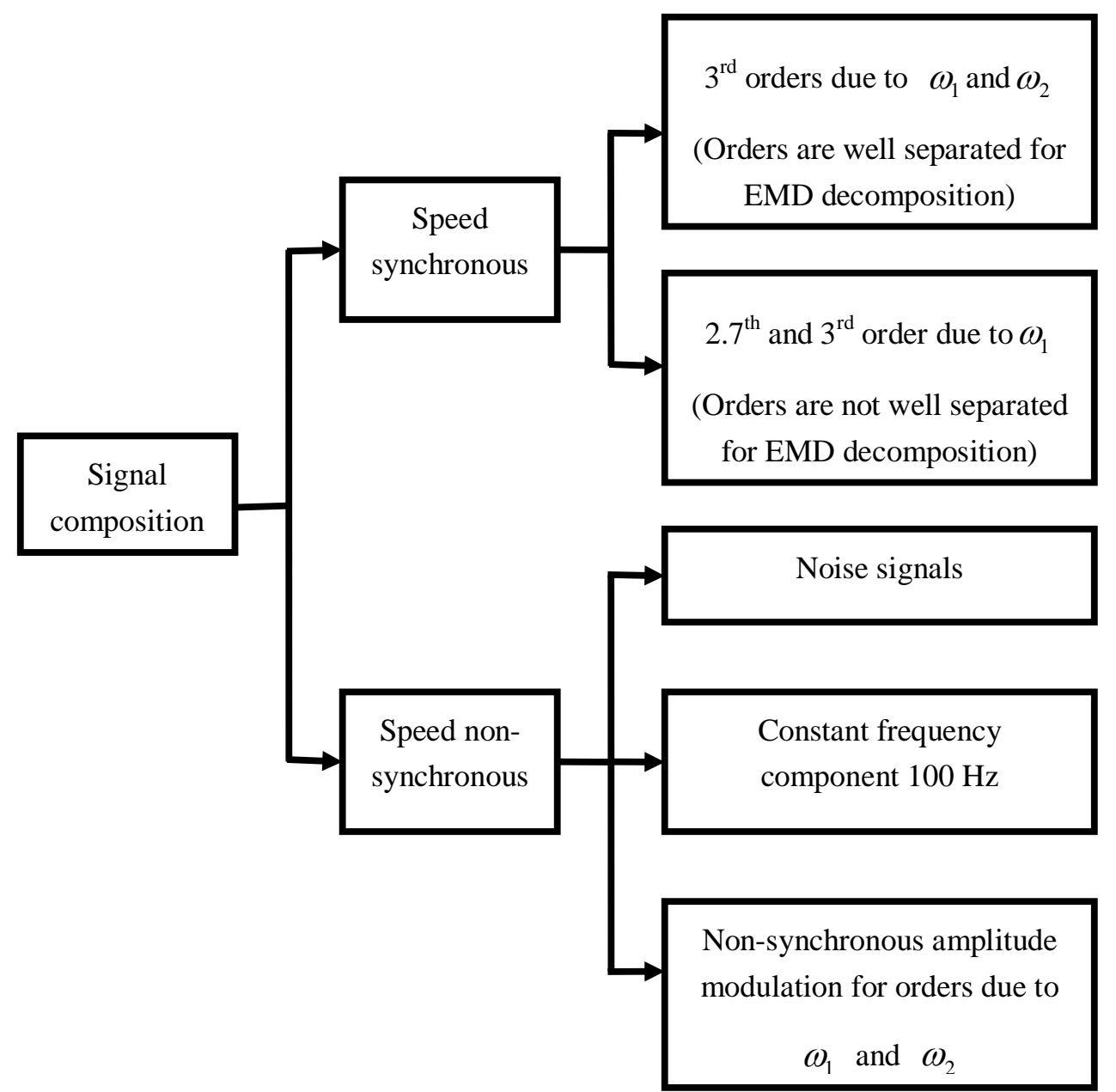

Figure 2: Signal composition of simulation study

Based upon the separation criterion shown in Figure 1, two cases are considered with the speed synchronous or order signals. The $3^{\text {rd }}$ orders due to rotating speed components $\omega_{1}$ and $\omega_{2}$ are well separated for EMD decomposition. The $2.7^{\text {th }}$ and $3^{\text {rd }}$ orders of $\omega_{1}$ are not suitable for decomposition by EMD. The amplitude and frequency relationships for these cases are also indicated in Figure 1. 
Among the speed non-synchronous signals, random noise is included since it is inevitable in reality, and a constant signal at $100 \mathrm{~Hz}$ is also added to simulate a typical multiple of electrical line frequency. For comparative purposes, speed synchronous amplitude and speed non-synchronous amplitude modulation for external excitation force are both simulated for comparison. The details are listed in Table 1. The Runge-Kutta-Gill method was used to obtain the system response in a MATLAB environment.

\subsection{Further decomposition of an IMF}

The calculated acceleration time response, the corresponding frequency spectrum and the RPM spectrum map are plotted in Figure 3 for displaying the nature of the signals by traditional signal processing methods. Where not specifically mentioned, all the figures are obtained for the speed non-synchronous amplitude modulation case.

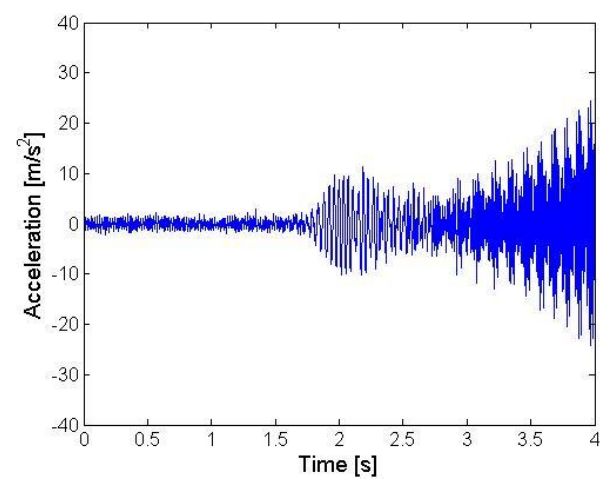

a. Time response

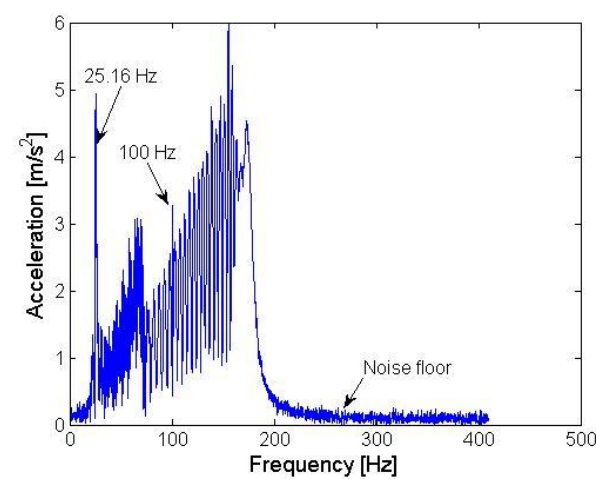

b. Frequency spectrum

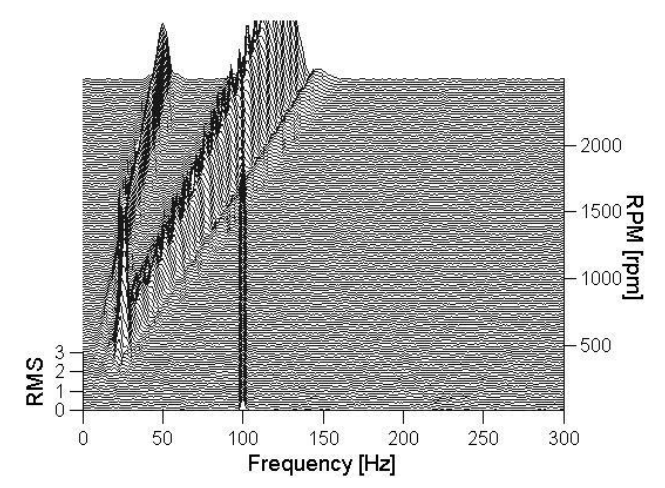

c. RPM spectrum map (based upon rotational speed $\omega_{1}$ )

Figure 3: Signal processing of raw signals

Figure 3(a) is the time response of the system. A clear beating effect of the signals can be seen which is due to the closely spaced sinusoidal external excitations. Figure 3(b) shows the signal in the frequency domain where a system resonance is present at 
$f_{n}=\frac{1}{2 \pi} \sqrt{k / m}=25.16 \mathrm{~Hz}$. The system resonance is due to the choice of the system parameters $m$ and $k$. This characteristic of the system is independent of the external forces. The smeared hump with the oscillating ripples is due to the quadratically increasing rotational speed, and the two sets of external forces that cause the high oscillating ripples over a wide range. The random noise floor is also seen in the spectrum. Further, the RPM spectrum map in Figure 3(c) shows how the signal evolves with speed. Two sets of speed varying signals can be observed and a clear 100 $\mathrm{Hz}$ component appears throughout the speed range and it is not influenced by speed. The traditional signal processing methods provide a clear understanding of the signal.

EMD may now be used to decompose the signals into IMFs, using the Hilbert Huang Transform Data Processing System (HHT-DPS1.4) obtained from NASA Goddard Space Flight Centre. The decomposition results are shown in Figure 4

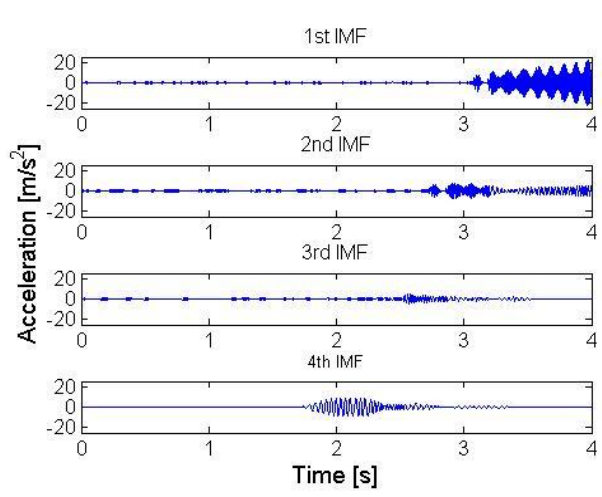

a. IMFs

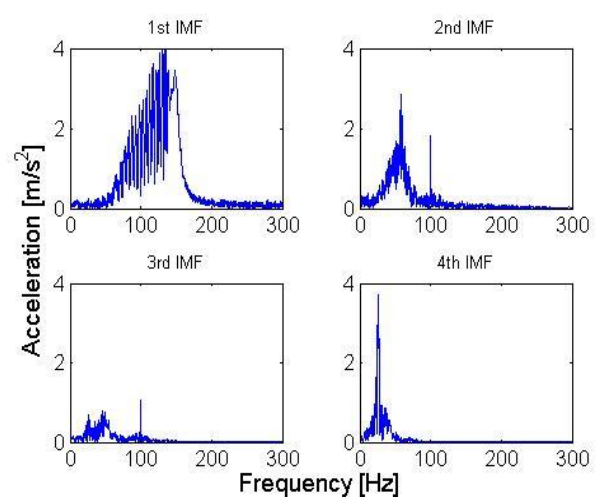

b. Frequency spectrum of IMFs

Figure 4: IMFs from EMD

Figure 4(a) indicates that the $1^{\text {st }}$ IMF also exhibit beating effects similar to that seen in Figure 3(a). Figure 4(b) shows the frequency spectra of these IMFs. The $1^{\text {st }}$ IMF spectrum shows a smeared hump with the oscillating ripples which indicates that it captures the system responses due to the closely spaced external forces. It is also clear that the system resonance is not included in the $1^{\text {st }}$ IMF. The $2^{\text {nd }}$ IMF also shows oscillating ripples in the spectrum in a lower frequency range, as well as a clear 100 $\mathrm{Hz}$ component. Clearly the oscillating ripples in Figure 3(b) are being separated into different IMFs and the system resonance is not evident in both $1^{\text {st }}$ and $2^{\text {nd }}$ IMFs. The $3^{\text {rd }}$ IMF again shows part of the oscillating ripples in the spectrum and also a $100 \mathrm{~Hz}$ component albeit much smaller than those in the $2^{\text {nd }}$ IMF. Lastly, the $4^{\text {th }}$ IMF clearly shows system resonance and it does suggest that the system resonance is being separated and specifically concentrated in the $4^{\text {th }}$ IMF. From these observations of the EMD results, it is found that the system resonance components, $100 \mathrm{~Hz}$ component as well as the oscillating ripples are separated into different IMFs. However the physical significance of the ripples in the different IMFs is still vague. 
Therefore order tracking techniques are now used to assist in the further clarification and decomposition of the signals. Computed order tracking (COT) is applied to the IMFs referring to $\omega_{1}$ in Figure 5. In this signal analysis, the observed signal from speed synchronous amplitude case is also plotted for comparison. The two computed order tracking results are shown in Figure 5(a) and (b). In order to compare order components in different IMFs, the highest order amplitude in the $1^{\text {st }}$ IMF is used to normalize all four order spectra maps so that the amplitude relationship between IMFs can be easily seen. (This normalized process is applied to all the following order domain analysis).
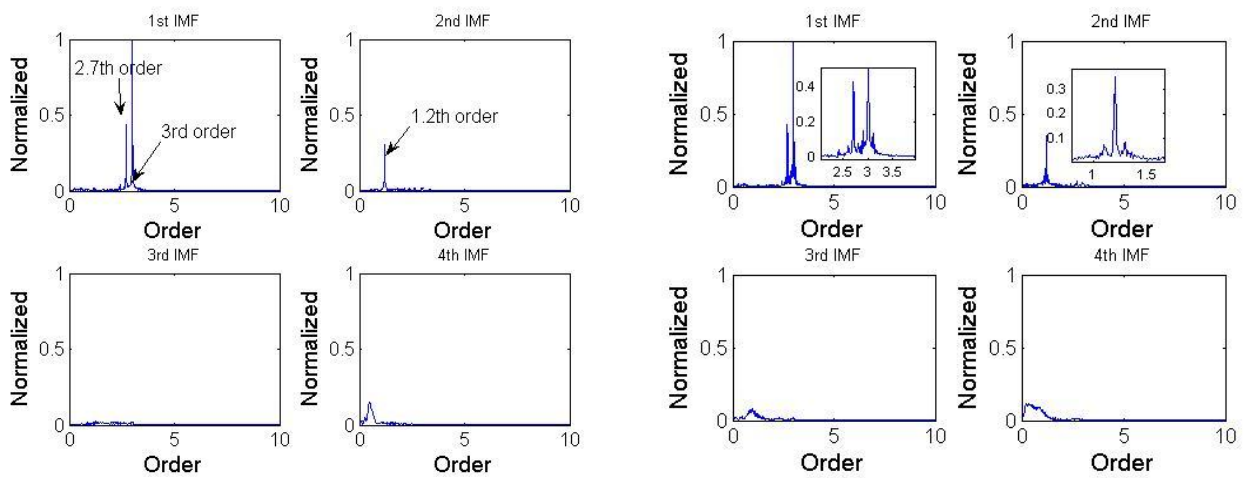

a. Speed synchronous amplitude

b. Speed non-synchronous amplitude

Figure 5: Normalized order spectra for IMFs

Looking at Figure 5(a) of the speed synchronous amplitude case, it is seen that the $1^{\text {st }}$ IMF clearly consists of the $2.7^{\text {th }}$ and $3^{\text {rd }}$ orders. The $2.7^{\text {th }}$ order is nearly half of $3^{\text {rd }}$ order in amplitude which corresponds well to the preset amplitude ratio of 0.5 in the force components. In the $2^{\text {nd }} I M F$ a $1.2^{\text {th }}$ order component can be seen. The $2^{\text {nd }} I M F$ indeed captures another rotating speed signal, since it corresponds very well to the speed ratio of 2.5 between the two speeds. However, compared with dominant $1^{\text {st }}$ IMF, this $1.2^{\text {th }}$ order is much smaller. Besides, it should still be remembered that in Figure 4(b), both $3^{\text {rd }}$ and $4^{\text {th }}$ IMFs show clear spectra in the frequency domain, especially for the $4^{\text {th }}$ IMF of its system resonance component. However since the re-sampling process of COT transforms equal time intervals to equal angular intervals, and then the Fourier analysis actually captures only the periodic signals with respect to angle instead of time (as is especially for the case of resonance), the system resonance and signals that non-synchronous with rotational speed, therefore, are de-emphasized once the re-sampling has been performed. As a result, the $3^{\text {rd }}$ and $4^{\text {th }}$ IMFs in the order spectra become much smaller than they are in frequency domain. This indicates that both $3^{\text {rd }}$ and $4^{\text {th }}$ IMFs are not closely related to rotational speed. Through COT analysis of IMFs in Figure 5(a), it may be concluded that the system responses due to the excitation at the two rotating speeds are well separated by EMD into the $1^{\text {st }}$ and $2^{\text {nd }}$ IMFs, however the close varying harmonics are not being separated in the $1^{\text {st }} I M F$. 
Then, the speed non-synchronous amplitude modulation case is studied in Figure 5(b). Firstly, compared to Figure 5(a), four similar resultant order maps are rendered. Again, EMD successfully separates the system responses due to the two rotating speeds into the $1^{\text {st }}$ and $2^{\text {nd }}$ IMFs. At the same time it can not further separate close varying harmonics in $1^{\text {st }}$ IMF. However upon closer inspection of the zoomed figures of the $1^{\text {st }}$ and $2^{\text {nd }}$ IMFs, sidebands appear in the order spectrum which do not occur in the speed synchronous amplitude case in Figure 5(a). This is due to the non-synchronous amplitude modulation of external excitation force. These signals are very important indications of system changes. However, they are quite small and easy to be neglected. In order to clearly visualize them, the zoomed figures are also plotted on top of the figures in Figure 5(b). Besides, with regard to these sidebands the traditional VKF-OT method can not perform the filtering properly since the sidebands are not synchronous with speed. Thus, it deserves further decomposition. The $1^{\text {st }}$ IMF is chosen for illustrating this further decomposition process.

The Vold-Kalman filter can thereafter be used for $1^{\text {st }}$ IMF at speed non-synchronous amplitude modulation case. Firstly, the dominant $3^{\text {rd }}$ order is extracted. A $20 \%$ relative filter bandwidth is used. This means that the ratio of the instantaneous absolute filter bandwidth to the instantaneous rotational speed frequency is 0.2. (See [15] and [16] for more details of the choice of the filter bandwidth). In the top figure of Figure 6(a), the spectra of the $1^{\text {st }}$ IMF and $3^{\text {rd }}$ order are superimposed together and therefore the relationship between them can be seen. The corresponding time waveform of $3^{\text {rd }}$ order is also plotted at the right hand side top figure of Figure 6(b). After the extraction of $3^{\text {rd }}$ order the rest of signal is also plotted in the frequency domain in the bottom figure of Figure 6(a). The $2.7^{\text {th }}$ order is also extracted and its corresponding time wave is plotted in Figure 6(b). Clearly the majority of signals are being extracted since $1^{\text {st }}$ IMF is a strongly speed related IMF.

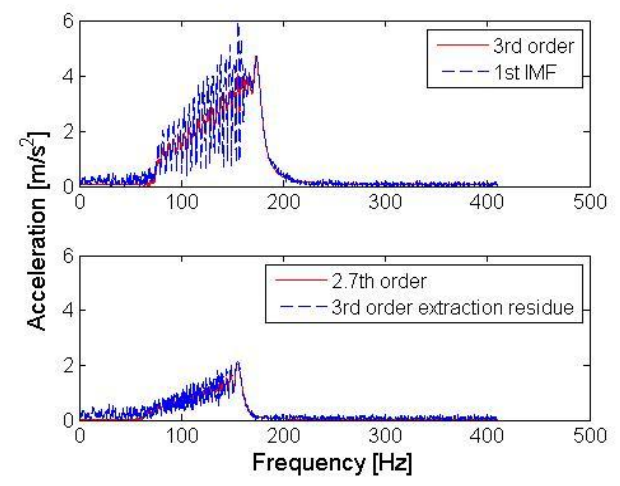

a. Spectrum maps

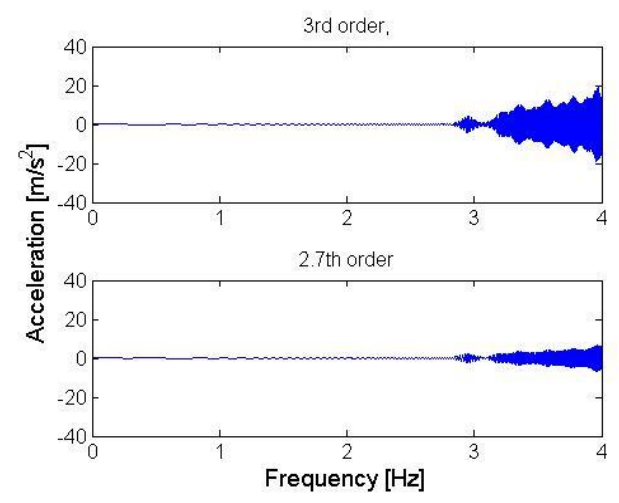

b. Time waveform

Figure 6: Separation of $2.7^{\text {th }}$ and $3^{\text {rd }}$ orders

With the help of computed order tracking to recognize orders and Vold-Kalman filter order tracking to extract them in time waveforms, the $2.7^{\text {th }}$ and $3^{\text {rd }}$ order signals that are synchronous with the rotational speed are successfully decomposed from the $1^{\text {st }}$ 
IMF. However, vibration signals that are non-synchronous with the rotational speed, especially for this case with amplitude modulation, should be further attended to. At this stage, if only VKF-OT were to be applied to the raw signals so that all the recognized orders are extracted through VKF-OT, the sidebands of the orders would be rendered and mixed together. However, instead of mixing this information, the combined IMF and VKF-OT provides the ability of focusing on the sidebands associated with the order of interest and distinguish them for analysis. This is a unique capability of the sequential use of EMD and order tracking methods. The results are plotted in Figure 7.

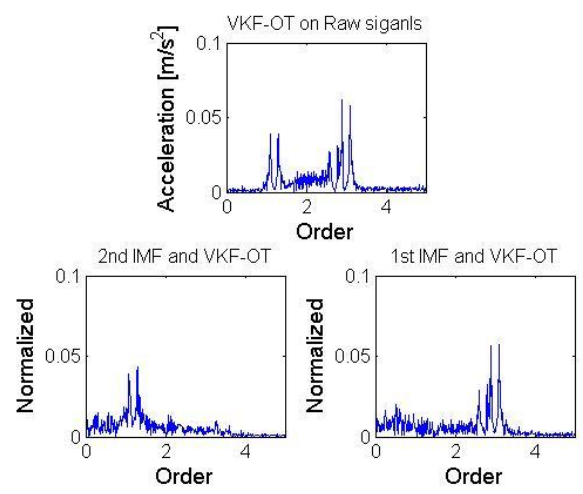

Figure 7: Separation of $1.2^{\text {th }}$ and $3^{\text {rd }}$ order sidebands

The top plot in Figure 7 is the order domain results of raw signals after subtracting the $1.2^{\text {th }}, 2.7^{\text {th }}$ and $3^{\text {rd }}$ orders by VKF-OT. It can be seen that two sets of sidebands of $1.2^{\text {th }}$ and $3^{\text {rd }}$ orders remain. Since the VKF-OT can not further extract non-synchronous amplitude modulation sidebands properly, therefore the traditional order tracking method stops here. However by using $1^{\text {st }}$ and $2^{\text {nd }}$ IMFs and VKF-OT, all recognized orders can be filtered out and renders separated sidebands into the bottom two figures of Figure 7 (Notice that $100 \mathrm{~Hz}$ component in $2^{\text {nd }}$ IMF can also be extracted by VKF-OT through considering it as a constant rotational speed at $R P M=100 \times 60=6000(\mathrm{rpm}))$. The bottom two figures achieve further separation of sidebands with the help of different IMFs. As a result, $1.2^{\text {th }}$ and $3^{\text {rd }}$ order sidebands are well separated into two figures which are intractable by traditional VKF-OT as is shown in the top figure of Figure 7. If further comparing the results of sequence use of two methods to EMD as well as VKF-OT alone around $3^{\text {rd }}$ order, it leads to Figure 8 . 


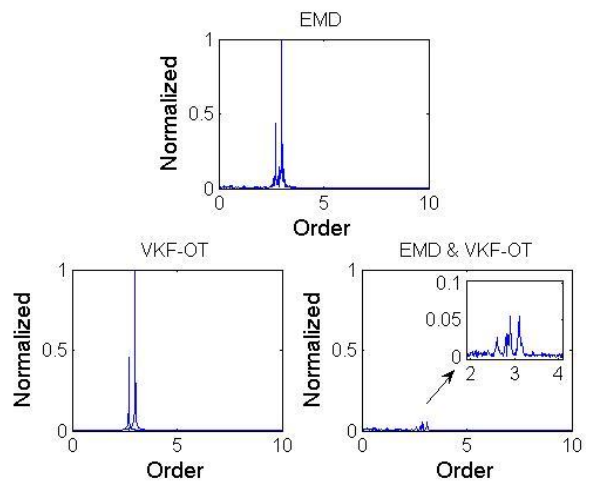

Figure 8: Results comparison of EMD, VKF-OT and sequence use of EMD \& VKF-OT (use $1^{\text {st }}$ IMF to illustrate)

For EMD result in Figure 8 on the top, the $1^{\text {st }}$ IMF captures both $2.7^{\text {th }}$ and $3^{\text {rd }}$ order as well as some sidebands around dominant orders. Clearly the amplitude modulation effects on sidebands are fairly small and are emaciated by the dominant orders. EMD can not further decompose this $1^{\text {st }}$ IMF. Through VKF-OT, the $2.7^{\text {th }}$ and $3^{\text {rd }}$ orders are properly extracted and superimposed together in the figure. However, no sideband information appears in the spectrum and therefore the ability of detecting the changes on amplitude modulation effects is lost. More importantly, since the amplitude modulation is non-synchronous with rotational speed, it is not amenable to VKF-OT. Through the sequential use of EMD and order tracking methods, the sideband information is however separated in the last figure of Figure 8. From Figure 8, it is clear that the amplitude modulation sidebands could not be extracted by using EMD or VKF-OT alone.

In the context of machine diagnostics, it should be emphasised that the un-extractable amplitude modulation effects by using either EMD or VKF-OT, are extracted through combining the two techniques in sequence. The extraction of amplitude modulation effects around the $3^{\text {rd }}$ order excludes the interference from the other order, i.e. $1.2^{\text {th }}$ order, so that analyst may focus on the amplitude variations of the $3^{\text {rd }}$ order. This makes the condition monitoring process more flexible and selective. In short, the sequential use of EMD and order tracking methods provides an advantage compared to EMD or VKF-OT in isolation. Information which would be un-extractable through both techniques alone can be achieved by combining them in sequence.

\section{Experimental study}

\subsection{Experimental test rig}

An experimental study was conducted to further illustrate the effectiveness of order tracking techniques in the decomposition of IMFs from EMD in an experimental environment. A laboratory-scale test rig comprising an automotive alternator was 
designed and constructed as shown in Figure 9(a). The resin on the outer isolation layer on one of the stator windings in the alternator was artificially damaged. This introduced an inter-turn short circuit between the windings, referred to in this study as the seeded fault. The original state of stator windings is shown in Figure 9(b).

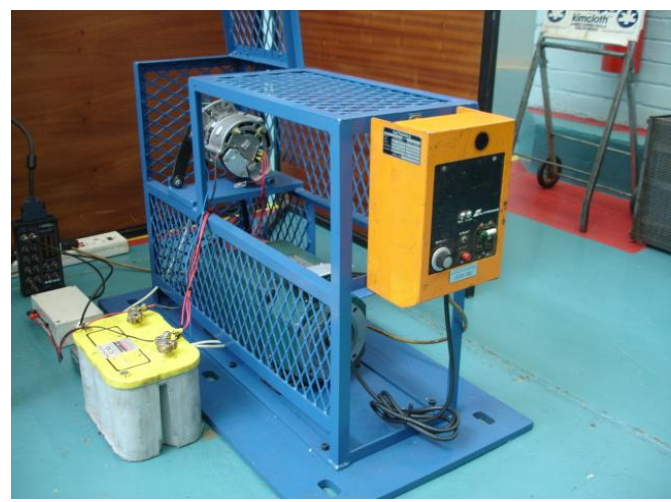

(a) Experimental test rig

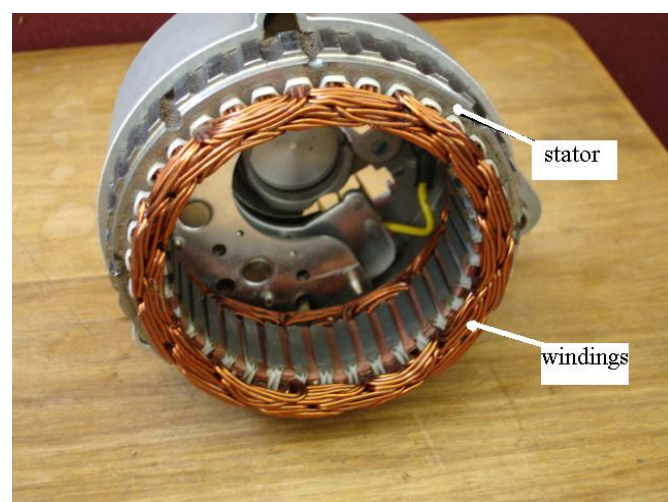

(b) Original state of stator windings

Figure 9: Experimental test rig and original state of stator windings

The main components constituting the test rig are the alternator, the variable speed motor, the controller and the battery. The alternator (which has 36 stator bars and 12 claws on the rotor) is driven by a variable speed induction motor via a V-belt. The 36 stator bars are shown in Figure 9(b) and the 12 rotor claws in Figure 10(a). The variable speed Saftronics motor (with a maximum speed of $1750 \mathrm{rpm}$ ) is controlled by a Saftronics DC controller (rated input $220 \mathrm{~V}$ and $15 \mathrm{~A}$ with a rated output of $180 \mathrm{~V}$ and $12 \mathrm{~A}$ ). The alternator charges an automotive battery which is used as the system load.

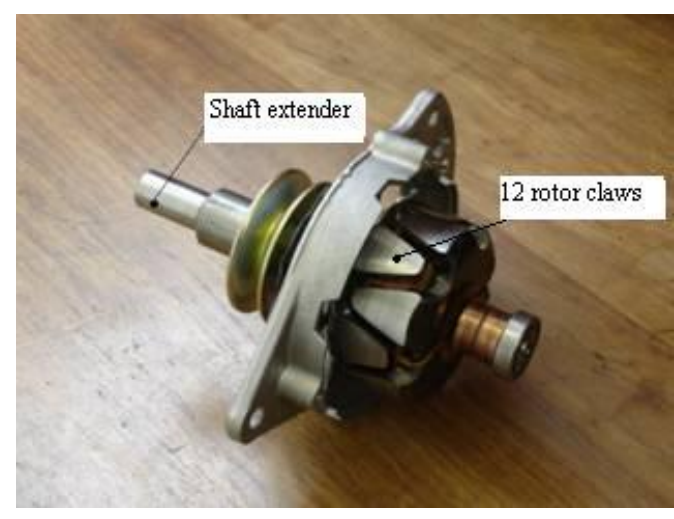

(a) Rotor claws and shaft extender

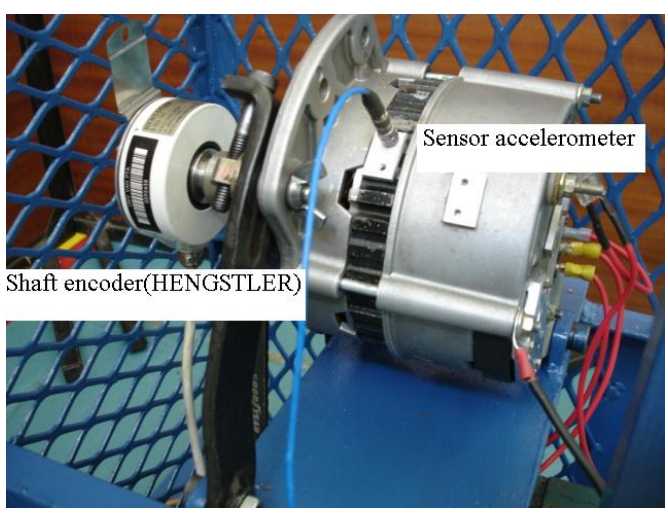

(b) Monitoring equipment

Figure 10: Signal sampling

The monitoring equipment for this test rig comprised an accelerometer (PCB Piezotronics M353B18 with sensitivity $10.13 \mathrm{mV} / \mathrm{g}$ and a frequency range $1 \mathrm{~Hz}-10$ $\mathrm{kHz}$ ) and a shaft encoder mounted on a specially made shaft extender (Hengstler RI 76 TD with 1-pulse revolution). They were assembled as shown in Figure 10(b). 
Signal sets were captured with a National Instruments board (NIB PCI-6024E with a sampling frequency of $10 \mathrm{kHz}$ for two-channel sampling) and transferred to a Pentium 4 computer for analysis. For further details of the test rig, refer to [17]. A typical data set obtained from the test rig is shown in Figure 11.
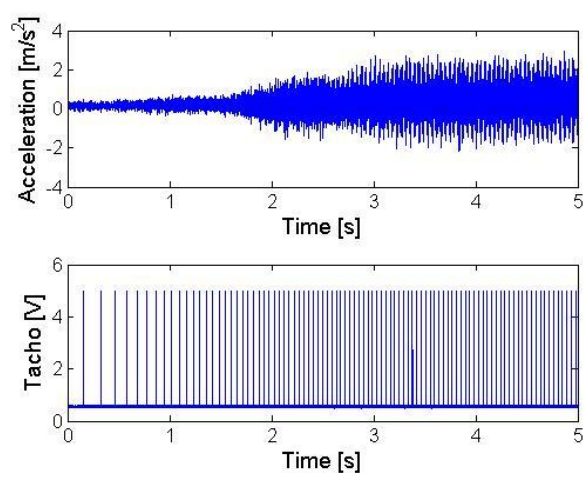

Figure 11: Typical experimental data

\subsection{Experimental signal analysis}

Firstly, a data set corresponding to the alternator in good condition was considered. Since there are 36 stator bars in the alternator, the $36^{\text {th }}$ order and its multiples are key features of the signal. Therefore, EMD and VKF-OT were used to focus on the $36^{\text {th }}$ order. The extracted $36^{\text {th }}$ order by VKF-OT is used to compare the EMD results. In order to identify which IMF captured the $36^{\text {th }}$ order, some basic frequency analysis was first performed. Firstly, a frequency analysis of the Vold-Kalman filter result for the $36^{\text {th }}$ order was done, and then a frequency analysis for each IMF was compared with the results from Vold-Kalman filter. In this way, it was recognized that, in this case, $6^{\text {th }}$ IMF captured most of the energy associated with the $36^{\text {th }}$ order. Figure 12 shows the results in the time domain as well as in the frequency domain.
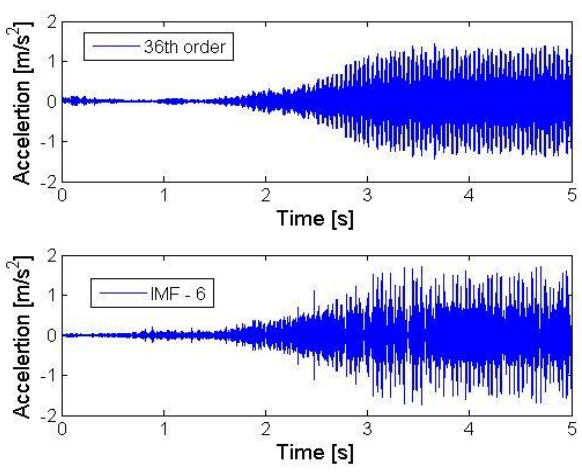

(a) Time domain waveforms
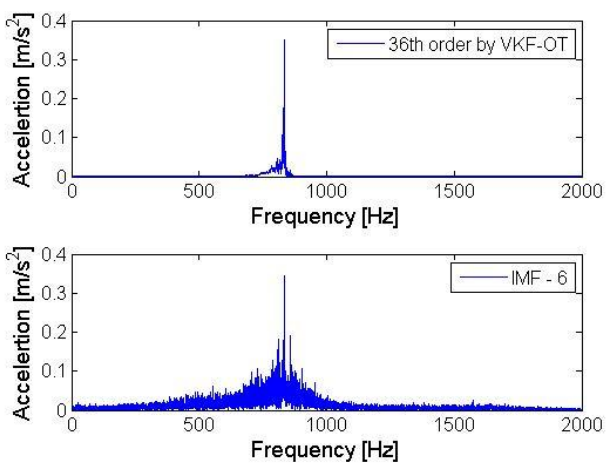

(b) Frequency domain spectrum

Figure 12: The $36^{\text {th }}$ order by VKF-OT and $6^{\text {th }}$ IMF

It can be seen from Figure 12(a) that roughly speaking, the $36^{\text {th }}$ order by VKF-OT and the $6^{\text {th }}$ IMF are more or less equivalent in amplitude and shape. Strictly speaking, the 
result from VKF-OT is much cleaner and more uniform than its counterpart $6^{\text {th }}$ IMF. This follows from the mathematical definition of Equation (1) in which the sinusoidal nature is impressed on the filtered order of VKF-OT. Furthermore, the basic frequency analysis in Figure 12(b) shows that both of the two time-domain waves capture the same fundamental frequency at around $830 \mathrm{~Hz}$. However, it should be noted that the $6^{\text {th }}$ IMF is obviously more complex than the $36^{\text {th }}$ order by VKF-OT. This complexity is due to the resolution of the intrinsic mode function, in that all the signals that modulate the $36^{\text {th }}$ order may be included, on condition that the final shape of the wave satisfies the requirements of an IMF. Clearly the $6^{\text {th }}$ IMF captures the majority of the $36^{\text {th }}$ order and the corresponding highest frequency peak coincides with the Vold-Kalman filter result. The inclusion of other frequency components in one IMF is one of the shortcomings of EMD when extracting a mono-order component in real applications. However, it is also due precisely to this attribute that IMF may capture machine fault signals that modulate the order of interest. This observation from Figure 12 points to the possible further decomposition of $36^{\text {th }}$ order related IMF and the decomposition of the signal may be useful for condition monitoring. Besides, it should also be borne in mind that the EMD process in fact separates the $36^{\text {th }}$ order together with vibrations that modulate it from other interference of orders, such as multiples of the $36^{\text {th }}$ order, like the $72^{\text {nd }}$ order. Therefore, all attention can now be focused on the modulated $36^{\text {th }}$ order.

From the experience gained in the simulation study, the computed order tracking results may help to recognize orders for signals. Therefore, in the following, COT was again used to transform all the analysis signals into the order domain. The $6^{\text {th }}$ IMF was then further decomposed. In Figure 13, the order domain results of the $6^{\text {th }}$ IMF, the Vold-Kalman filter for the $36^{\text {th }}$ order from $6^{\text {th }}$ IMF and the sequential use of the two techniques are presented. 


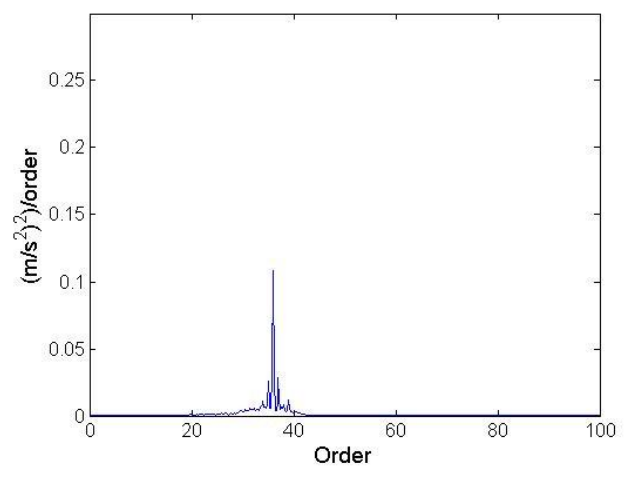

(a) $6^{\text {th }}$ IMF

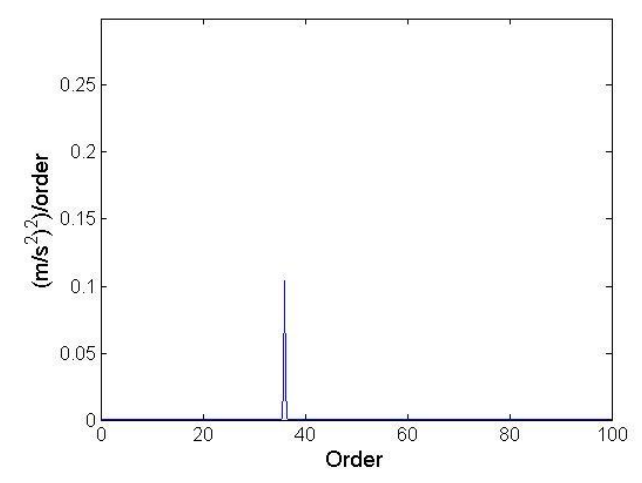

(b) Vold-Kalman filter order 36

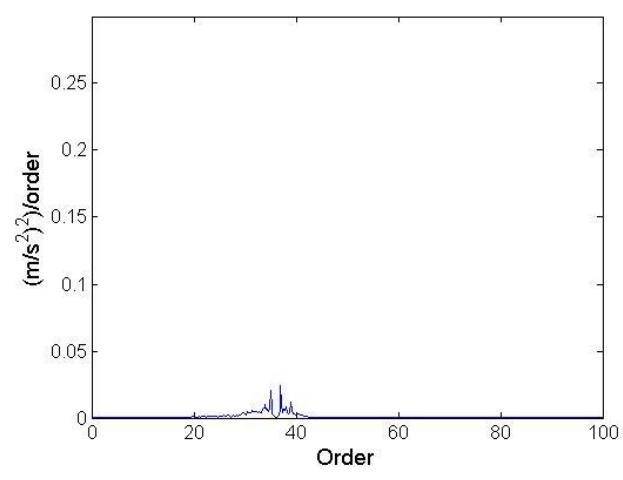

(c) Residue signal

Figure 13: PSD on $6^{\text {th }}$ IMF, Vold-Kalman filter $36^{\text {th }}$ order from $6^{\text {th }}$ IMF and residue signal in the order domain (good condition)

It is clear from Figure 13(a) that the $6^{\text {th }}$ IMF includes the $36^{\text {th }}$ order and some sidebands. After applying the Vold-Kalman filter, the $36^{\text {th }}$ order is clearly extracted from the $6^{\text {th }}$ IMF as shown in Figure 13(b). Figure 13(b) suggests that the Vold-Kalman filter succeeded in extracting the $36^{\text {th }}$ order from the $6^{\text {th }}$ IMF using a $20 \%$ relative filter bandwidth. (For details on the choice of the filter bandwidth, again refer to [15] and [16]). Then the residue signal of after sequential use of the two techniques contains the sidebands of the $36^{\text {th }}$ order, as is depicted in the order domain as shown in Figure 13(c). Based upon the order domain analysis of the $6^{\text {th }}$ IMF, it is clear that this IMF is dominated by $36^{\text {th }}$ order signal. Only some sidebands are included. There are no other prominent orders appearing around the $36^{\text {th }}$ order and therefore no need to further extract other orders. Besides, as was learnt from the simulation studies, amplitude modulation may cause sidebands around dominant orders. This is a very useful indication of machine condition. Thus, the residue signals of $6^{\text {th }}$ IMF which hold sidebands information will be useful for condition monitoring. This information cannot be extracted by traditional VKF-OT, but through further decomposition of $6^{\text {th }}$ IMF. For illustration, the time domain residue signal is also plotted in Figure 14(a) for this good condition data. 


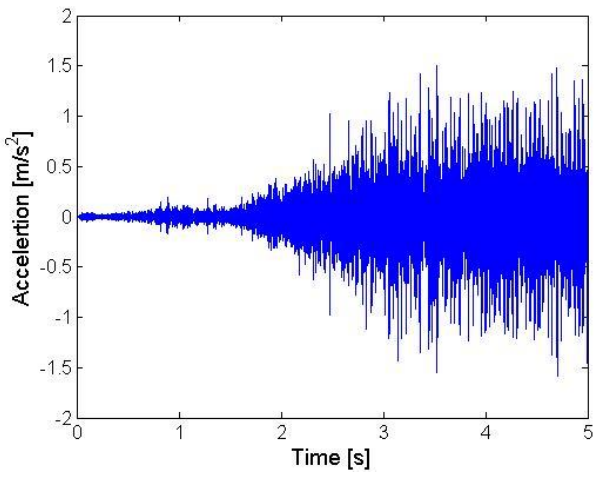

(a) Residue signal (good condition)

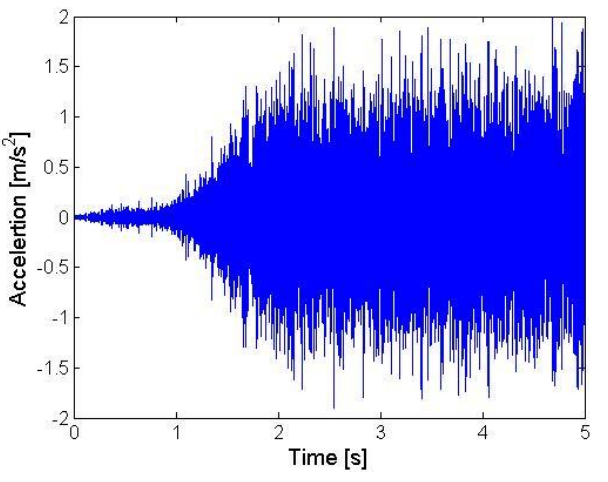

(b) Residue signal (bad condition)

Figure 14: Residue signal in good and fault condition

The seeded fault signal is subsequently considered. The same COT analysis was again performed for $6^{\text {th }}$ IMF, the $36^{\text {th }}$ order by VKF-OT from $6^{\text {th }}$ IMF and the results of combing the two techniques are shown in Figures 15(a), (b) and (c).

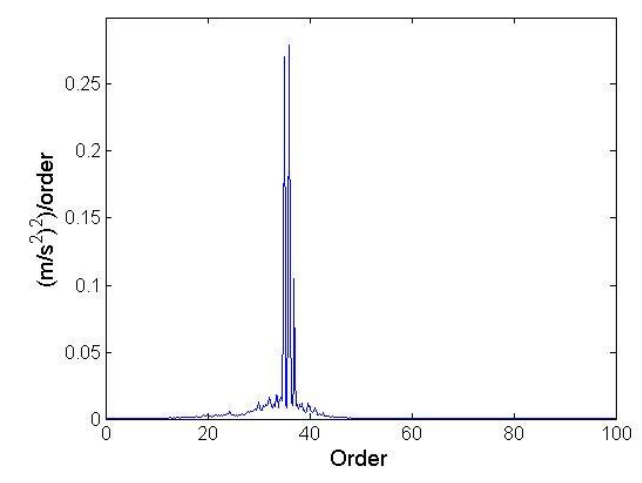

(a) IMF -6

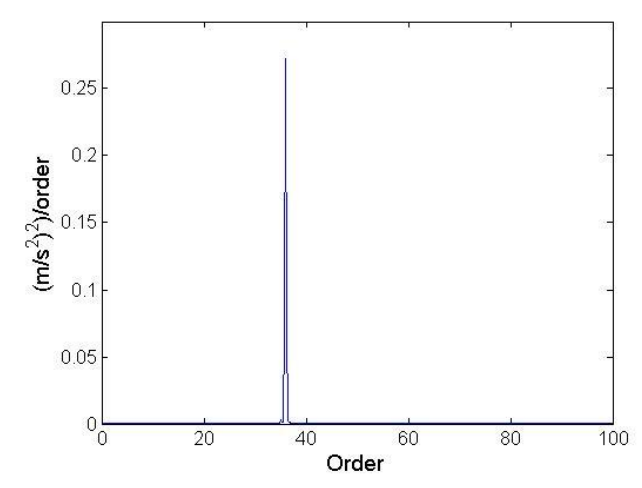

(b) Vold-Kalman filter order 36

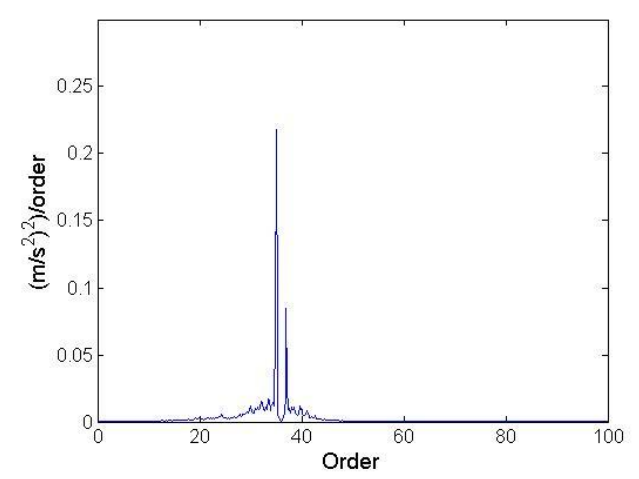

(c) Residue signal

Figure 15: PSD on IMF-6, Vold-Kalman filter $36^{\text {th }}$ order from IMF-6 and residue signal in order domain (seeded fault condition)

Due to the seeded fault, the amplitudes of the order component peaks in all three plots 
of Figure 15 have now clearly increased compared to the good condition shown in Figure 13. This is to be expected because the short circuit between the stator windings changes the electromagnetic field and causes changes in the vibration amplitude. This is especially the case for the $36^{\text {th }}$ order, which corresponds to the 36 stator bars in the alternator. Clearly, Figure 15 again shows the successful decomposition of $6^{\text {th }}$ IMF. The corresponding time-domain residue signal is also plotted in Figure 14(b). When compared with its counterpart in Figure 14(a), the system changes can be clearly identified simply from the amplitude of the two residue time-domain signals. Besides, the order domain peaks in Figures 15(b) and (c) have both substantially increased.

However, most importantly, with the help of order tracking techniques, $6^{\text {th }}$ IMF has been further decomposed into $36^{\text {th }}$ order and sidebands residues. This kind of separated vibrations is not achievable by EMD method or VKF-OT alone. Thus, the $36^{\text {th }}$ order as well as its sidebands information is available and it is worthwhile studying each part of the signal to track the system changes in this real case and see how the different parts of signals contribute to the overall changes. Therefore, one of the most frequently used time-domain techniques, namely root mean square (RMS) analysis, was used here to demonstrate the diagnostic ability of time-domain signals of different parts. Three RMS values were compared for good and seeded fault conditions, as shown in Table 2.

Table 2: RMS for different signals

\begin{tabular}{|l|l|l|c|}
\hline Signals & $\begin{array}{l}\text { RMS } \\
\text { good }\end{array}$ & $\begin{array}{l}\text { RMS } \\
\text { seeded fault }\end{array}$ & $\frac{(\text { RMS }(\text { fault })-R M S(\text { good }))}{R M S(\text { good })}$ \\
\hline IMF-6 & 0.3244 & 0.5758 & $77.5 \%$ \\
\hline $36^{\text {th }}$ order from IMF-6 & 0.1568 & 0.2686 & $71.3 \%$ \\
\hline Residue & 0.2770 & 0.4974 & $79.57 \%$ \\
\hline
\end{tabular}

It can be concluded from this table that the changes in $6^{\text {th }}$ IMF are caused by both the $36^{\text {th }}$ order and its residue. Clearly the RMS values of all of these signals change substantially. This result is reasonable, since the seeded fault in the inter-turn short circuit in the stator winding would influence the vibrations of both the $36^{\text {th }}$ order and its sidebands. An inter-turn short in the stator windings of an alternator would not introduce simple parameter changes in the system but influences the electromagnetic forces between stator and rotor in that entire area so that the vibration amplitude in that area is disturbed. Consequently, it should be expected that both order signals and sideband signals would be influenced by the introduction of the seeded fault. However, the further decomposition of $6^{\text {th }}$ IMF enables detailed studies of $36^{\text {th }}$ order and its sidebands vibrations in time domain. For this specific case, changes in the sidebands residue signal were higher than the changes in the $36^{\text {th }}$ order, by $79.57 \%$ and $71.3 \%$ respectively. Both of these signals are indicators of the stator inter-turn short. 
However, different part of signals may indicate the changes of different scenarios of machine conditions. When both the $36^{\text {th }}$ order and its sidebands increase evenly, it means the whole electromagnetic force is increased, whereas, if additional sidebands increase compared to the dominant $36^{\text {th }}$ order, this means that more amplitude modulation occurs.

Thus, from these detailed studies of the signals, it may be deduced that, after introduction of the seeded fault, the amplitude modulations of the $36^{\text {th }}$ order become more severe than for the good condition. This is a clear indication of a machine inter-turn short which causes the change of electromagnetic force in a certain area and result in amplitude modulation of vibrations in the $36^{\text {th }}$ order. As a result, the further decomposition of $6^{\text {th }}$ IMF in this experimental study provides a method to examine in detail each part of the signal so as to provide in-depth understanding of machine condition.

The experimental study therefore demonstrates that order tracking (COT and VKF-OT) may be employed to further decompose the IMFs and therefore provides additional diagnostic capabilities for distinguishing and evaluating fault severity in machine vibration signals.

\section{Conclusion}

For rotating machinery, both order signals and vibrations that modulate orders are important machine condition indicators. Order signals are strictly synchronous with rotational speed and can be extracted by order tracking methods, such as Vold-Kalman filter order tracking. Vibrations that modulate orders, however, may be synchronous or non-synchronous with rotational speed and are therefore usually difficult to extract by order tracking alone. However, empirical mode decomposition usually includes these vibrations with the orders into different intrinsic mode functions. Therefore, further decomposition of intrinsic mode functions through order tracking is proposed in this paper. Using this approach, vibrations that modulate orders which are useful for condition monitoring have been extracted in both simulation and experimental studies. Identification of these vibrations is not achievable through empirical mode decomposition or traditional order tracking methods alone. Both the simulation studies and the experimental tests therefore support the further idea of decomposing intrinsic mode functions for condition monitoring.

\section{Acknowledgement}

The authors gratefully acknowledge NASA Goddard Space Flight Centre that granted permission to use their toolkit: Hilbert Huang Transform Data Processing System (HHT-DPS1.4). 


\section{References:}

[1] Huang, N. E., Shen, Z., Long, S. R., Wu, M. C., Shin, H. H., Zheng, Q., Yen, N. C., Tung, Ch. Ch. and Liu, H. H., The empirical mode decomposition and the Hilbert spectrum for nonlinear and non-stationary time series analysis, in: Proceedings of the Royal Society, London, 454, 1998, pp.903-995.

[2] Guo, D. and Peng, Z. K., Vibration analysis of a cracked rotor using Hilbert-Huang transform, Mechanical Systems and Signal Processing, 2007, 21, pp.3030-3041.

[3] Chen, H. G., Yan, Y. J. and Jiang, J. S., Vibration-based damage detection in composite wingbox structures by HHT, Mechanical Systems and Signal Processing, 2007, 21, pp.307-321.

[4] Liu, B., Riemenschneider, S. and $\mathrm{Xu}, \mathrm{Y} .$, Gearbox fault diagnosis using empirical mode decomposition and Hilbert spectrum, Mechanical Systems and Signal Processing, 2006, 20, pp.718-734.

[5] Cohen, L., Time-frequency analysis, Englewood Cliffs, NJ: Prentice-Hall.

[6] Schwartz, M., Bennett, W. R. and Stein, S., Communications systems and techniques, New York: McGraw-Hill.

[7] Flandrin, P., Rilling, G. and Gonçalvés, P., Empirical mode decomposition as a filter bank, IEEE Signal Processing Letters, 11, No. 2, February 2004.

[8] Eggers, B. L., Heyns, P. S. and Stander, C. J., Using computed order tracking to detect gear condition aboard a dragline, The Journal of Southern African Institute of Mining and Metallurgy, 2007,107 , pp.1-8.

[9] Gao, Q., Duan, C., Fan, H. and Meng, Q., Rotating machine fault diagnosis using empirical mode decomposition, Mechanical Systems and Signal Processing, 2008, 22, pp.1072-1081.

[10] Wu, F. J., Qu, L. S., Diagnosis of subharmonic faults of large rotating machinery based on EMD, Mechanical Systems and Signal Processing, 2009,23, pp.467-475.

[11] Blough, J. R., A survey of DSP methods for rotating machinery analysis, what is needed, what is available, Journal of Sound and Vibration, 2003, 262, pp.707-720.

[12] Yang, Zh. J., Yang , L. H., Qing, Ch. M. and Huang, D., A method to eliminate riding waves appearing in the empirical AM/FM demodulation, Digital Signal Processing, 2008, 18, pp.488-504.

[13] Feldman, M., Analytical basics of the EMD: Two harmonics decomposition, Mechanical System and Signal Processing, 2009, 23, pp.2059-2071.

[14] Stoker, J. J., Nonlinear vibrations in mechanical and electrical systems, New York: Wiley, London: Chapman and Hall: 1948, 1992.

[15] Wang, K. and Heyns, P. S., Vold-Kalman filter order tracking in vibration monitoring of electrical machines, Journal of Vibration and Control, 2009, 15(9), pp.1325-1347.

[16] Herlufsen, H., Gade, S. and Konstantin-Hansen, H., Characteristics of the 
Vold/Kalman order tracking filter, Proceedings of the $17^{\text {th }}$ International Modal Analysis Conference, Kissimmee, FL, February 8-11, 1999.

[17] Wang, K., Vibration monitoring on electrical machine using Vold-Kalman Filter Order Tracking. Dissertation for the degree: Master of Science.

Available at:

http://upetd.up.ac.za/thesis/available/etd-08282008-171945/unrestricted/disse rtation.pdf

[Accessed on: 8 Sep. 2009] 